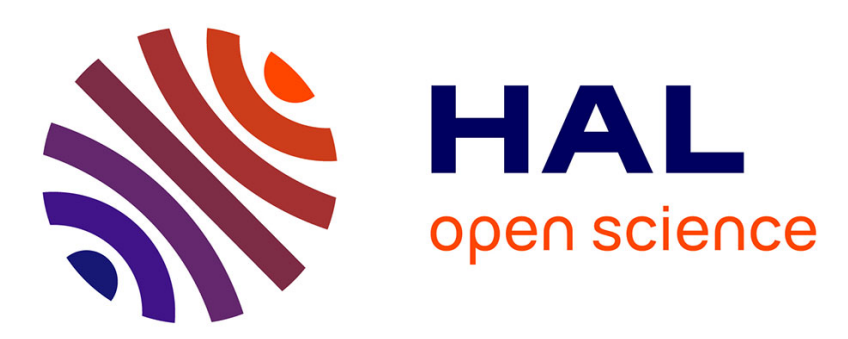

\title{
Timestepping schemes for nonsmooth dynamics based on discontinuous Galerkin methods: Definition and outlook
}

\author{
Thorsten Schindler, Vincent Acary
}

\section{To cite this version:}

Thorsten Schindler, Vincent Acary. Timestepping schemes for nonsmooth dynamics based on discontinuous Galerkin methods: Definition and outlook. Mathematics and Computers in Simulation, 2014, 95, pp.180-199. 10.1016/j.matcom.2012.04.012 . hal-00762850

\section{HAL Id: hal-00762850 \\ https://hal.inria.fr/hal-00762850}

Submitted on 28 Oct 2017

HAL is a multi-disciplinary open access archive for the deposit and dissemination of scientific research documents, whether they are published or not. The documents may come from teaching and research institutions in France or abroad, or from public or private research centers.
L'archive ouverte pluridisciplinaire HAL, est destinée au dépôt et à la diffusion de documents scientifiques de niveau recherche, publiés ou non, émanant des établissements d'enseignement et de recherche français ou étrangers, des laboratoires publics ou privés. 


\title{
Timestepping schemes for nonsmooth dynamics based on discontinuous Galerkin methods: Definition and outlook
}

\author{
Thorsten Schindler ${ }^{\mathrm{a}, *}$, Vincent Acary ${ }^{\mathrm{b}}$ \\ ${ }^{a}$ Technische Universität München, Boltzmannstraße 15, 85748 Garching, Germany \\ b INRIA 655 avenue de l'Europe, Montbonnot, 38334 Saint Ismier Cedex, France
}

The contribution deals with timestepping schemes for nonsmooth dynamical systems. Traditionally, these schemes are locally of integration order one, both in non-impulsive and impulsive periods. This is inefficient for applications with infinitely many events but large non-impulsive phases like circuit breakers, valve trains or slider-crank mechanisms. To improve the behaviour during non-impulsive episodes, we start activities twofold. First, we include the classic schemes in time discontinuous Galerkin methods. Second, we split non-impulsive and impulsive force propagation. The correct mathematical setting is established with mollifier functions, Clenshaw-Curtis quadrature rules and an appropriate impact representation. The result is a Petrov-Galerkin distributional differential inclusion. It defines two Runge-Kutta collocation families and enables higher integration order during non-impulsive transition phases. As the framework contains the classic Moreau-Jean timestepping schemes for constant ansatz and test functions on velocity level, it can be considered as a consistent enhancement. An experimental convergence analysis with the bouncing ball example illustrates the capabilities.

Keywords: Timestepping scheme; High order; Nonsmooth dynamics; Time discontinuous Galerkin methods; Impact

\section{Notation}

The following notation is used throughout the paper. Let $I$ denote a real time interval. A function $f: I \rightarrow \mathbb{R}^{n}$ is said to be of class $\mathcal{C}^{p}\left(I ; \mathbb{R}^{n}\right)$ if it is continuously differentiable up to the order $p$. The set of functions $f: I \rightarrow \mathbb{R}^{n}$ that are absolutely continuous on $I$ is denoted by $\mathcal{W}^{1,1}\left(I ; \mathbb{R}^{n}\right)$. The set of functions $f: I \rightarrow \mathbb{R}^{n}$ that are locally Lebesgue integrable on $I$ is referred to as $L_{\text {loc }}^{1}\left(I ; \mathbb{R}^{n}\right)$. The set of functions $f: I \rightarrow \mathbb{R}^{n}$ of bounded variations (BV) is represented by $\mathcal{B} \mathcal{V}\left(I ; \mathbb{R}^{n}\right)$. For $f \in \mathcal{B} \mathcal{V}\left(I ; \mathbb{R}^{n}\right)$, the right-limit function is given by $f^{+}(t)=\lim _{s \rightarrow t, s>t} f(s)$, and respectively the leftlimit function by $f^{-}(t)=\lim _{s \rightarrow t, s<t} f(s)$. The jump of $f$ at $t$ is symbolized by $[[f(t)]]=f^{+}(t)-f^{-}(t)$. The set of functions $f: I \rightarrow \mathbb{R}^{n}$ of locally bounded variations (LBV) is expressed as $\mathcal{L B V}\left(I ; \mathbb{R}^{n}\right)$. In all cases, we skip the image space if there is no ambiguity and we extend the domain if necessary.

\footnotetext{
* Corresponding author.

E-mail addresses: thorsten.schindler@mytum.de (T. Schindler), vincent.acary@inria.fr (V. Acary).

URLs: http://www.amm.mw.tum.de/ (T. Schindler), http://www.inrialpes.fr/bipop/ (V. Acary).
} 
The set of measures on the interval $I$ is represented by $\mathcal{M}(I)$. We associate with any function $f \in \mathcal{L B} \mathcal{V}(I)$ a differential measure $\mathrm{d} f \in \mathcal{M}(I)$ [18]. The notation $\mathrm{d} t$ defines the Lebesgue measure on $\mathbb{R}$. The space of all realvalued, $\mathcal{C}^{\infty}$-functions with compact support in $I$ is denoted by $\mathcal{D}(I)$. The set of linear functionals that maps $\mathcal{D}(I)$ onto the set of real numbers defines the dual space $\mathcal{D}^{*}(I)$, which is called the space of distributions. For a distribution $d \in \mathcal{D}^{*}(I)$, it is conventional to write

$$
d: \mathcal{D}(I) \rightarrow \mathbb{R}, \quad \varphi \mapsto\langle d, \varphi\rangle
$$

where $\langle\cdot, \cdot\rangle$ is the primal-dual pairing and $\langle d, \cdot\rangle$ is the linear functional which defines $d$. For $f \in L_{\text {loc }}^{1}\left(I ; \mathbb{R}^{n}\right)$ (respectively a measure $\mu \in \mathcal{M}(I)$ ), a corresponding distribution $T_{f}$ (respectively $T_{\mu}$ ) is associated such that

$$
\left\langle T_{f}, \varphi\right\rangle=\int_{I} f \varphi \mathrm{d} t \quad\left(\text { respectively }\left\langle T_{\mu}, \varphi\right\rangle=\int_{I} \varphi \mu\right) .
$$

One abuses notation by identifying $T_{f}$ with $f$, i.e. $\langle f, \varphi\rangle=\left\langle T_{f}, \varphi\right\rangle$ (respectively $T_{\mu}$ with $\mu,\langle\mu, \varphi\rangle=\left\langle T_{\mu}, \varphi\right\rangle$ ). The distributional derivative of a distribution $d$ will be symbolized by $D d$ and is usually defined by

$$
\langle D d, \varphi\rangle:=-\langle d, \dot{\varphi}\rangle, \quad \forall \varphi \in \mathcal{D}(I) .
$$

We denote by $0=: t_{0}<t_{1}<\cdots<t_{k}<\cdots<t_{N}:=T$ a finite partition (or a subdivision) of the time interval $[0, T](T>0)$. The integer $N$ stands for the number of time intervals in the subdivision. The $N$ sub-intervals $I_{i}:=\left(t_{i-1}, t_{i}\right)$ are of length $\Delta t_{i}$ and define the time-steps. The time step-size partition is referred to as $\mathcal{I}:=\left\{I_{1}, \ldots, I_{N}\right\}$. The set of piecewise continuously differentiable functions on this subdivision is given by $\mathcal{C}^{p}\left(\mathcal{I} ; \mathbb{R}^{n}\right)$. The value of a real function $x(t)$ at the time $t_{k}$ is approximated by $x_{k}$.

\section{Point of departure}

This article treats higher order timestepping schemes based on time discontinuous Galerkin methods in the context of nonsmooth dynamics. We give a short introduction of nonsmooth dynamical systems in mechanics, of classical time integration schemes and of present strategies to achieve higher integration order during non-impulsive episodes.

\subsection{Nonsmooth dynamical systems}

The bouncing ball (cf. Fig. 1) is a typical nonsmooth dynamical system in the field of mechanics [29,10,6,24,16,2,26]. Informally, we can envisage the physical evolution as follows. During a finite time interval $\emptyset \neq I:=(0, T) \subset \mathbb{R}$, a ball with mass $m$ falls from an initial position $q_{0}$, given an initial velocity $v_{0}$ and some external momentum flow fdt. It hits the ground and lifts off again or stays calm depending on the resulting interaction di being partly elastic or plastic. If the impact events accumulate in finite-time, the first case is called a Zeno phenomenon if bouncing and free flight alternate infinitely often in $I$.

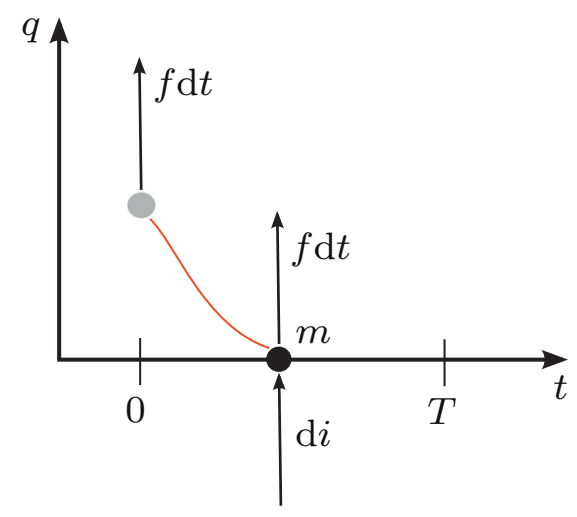

Fig. 1. Bouncing ball example. 
The most important realisation is the occurrence of a velocity jump due to the impact. The function describing the state of position and velocity contains non-impulsive and impulsive propagation episodes. Using a description based on classical function derivatives, one has to distinguish these two ranges and gets the following structure:

$$
\begin{aligned}
& q(0):=q_{0} \in \mathbb{R}, \\
& v(0):=v_{0} \in \mathbb{R}, \\
& \dot{q}=v \text { a.e., } \\
& \dot{v}=m^{-1} f+m^{-1} r \text { a.e., } \\
& v_{j}^{+}=v_{j}^{-}+m^{-1} p_{j} .
\end{aligned}
$$

Eq. (7) describes non-impulsive motion almost everywhere (a.e.) with the contact force $r$, whereas (8) defines impacts for countable time instances $t_{j}$. With appropriate mathematical objects, i.e. measures, it is possible to enter the modern theory of nonsmooth dynamical systems $[25,19,17,4]$. Problem 1.1 defines a consistent generalisation of the bouncing ball example, not distinguishing between non-impulsive and impulsive motion.

Problem 1.1 ((Measure differential inclusion)). Solve the initial value problem

$$
\begin{aligned}
\mathrm{d} q & =v \mathrm{~d} t, \\
\mathrm{~d} v & =m^{-1} f \mathrm{~d} t+m^{-1} \mathrm{~d} i
\end{aligned}
$$

in terms of measures together with the initial conditions (4) and (5).

Problem 1.1 is based on the following assumptions which let us rediscover (4)-(8).

- $q \in \mathcal{W}^{1,1}(I)$ is the absolutely continuous position with the measure $\mathrm{d} q=\dot{q} \mathrm{~d} t$ and the weak time derivative $\dot{q}$, i.e. the classical derivative almost everywhere according to Rademacher's theorem.

- $v \in \mathcal{L B V}(I)$ is the velocity of locally bounded variation. Omitting the Cantor part of the singular measure, one can split its associated measure $\mathrm{d} v \in \mathcal{M}(I)$

$$
\mathrm{d} v:=\gamma \mathrm{d} t+\sum_{j}\left[\left[v_{j}\right]\right] \delta_{t_{j}}=\gamma \mathrm{d} t+\sum_{j}\left(v_{j}^{+}-v_{j}^{-}\right) \delta_{t_{j}}
$$

in a locally integrable (non-impulsive) and atomic (impulsive) part with

accelerations $\gamma \in L_{\text {loc }}^{1}(I)$,

countable velocity jumps $v_{j}^{ \pm} \in \mathbb{R}$ and Dirac measures $\delta_{t_{j}}$.

- $0<m^{-1}:=m^{-1}(q) \in \mathcal{C}^{0}(\mathbb{R})$ is the inverse mass.

- $f:=f(t, q, v) \in L_{\text {loc }}^{1}(I \times \mathbb{R} \times \mathbb{R} ; \mathbb{R})$ is an external force.

- $i \in \mathcal{L B V}(I)$ is the interaction (impulse) of locally bounded variation. Omitting the Cantor part of the singular measure, one can split its associated measure

$$
\mathrm{d} i:=r \mathrm{~d} t+\sum_{j} p_{j} \delta_{t_{j}}
$$

being part of

contact relations $(q, v, r, t) \in \mathcal{N}_{C}$,

countable impact relations $\left(q_{j}, v_{j}, p_{j}, t_{j}\right) \in \mathcal{N}_{I}$.

The inclusions (15) and (16) are formal ways to state contact relations or other nonsmooth laws. We can write the contact force $r:=r(t, q, v) \in L_{\text {loc }}^{1}(I \times \mathbb{R} \times \mathbb{R} ; \mathbb{R})$ as a locally integrable function. In practice, this might not always be obvious. If the contact relation is single-valued, the contact force is a (compliant) function of position $q$, velocity $v$ and time $t$. However, if the contact relation is set-valued, one has to solve nonlinear/nonsmooth relations to gain $r$. 


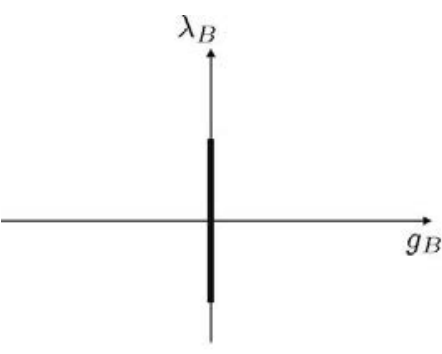

(a) Bilateral contact

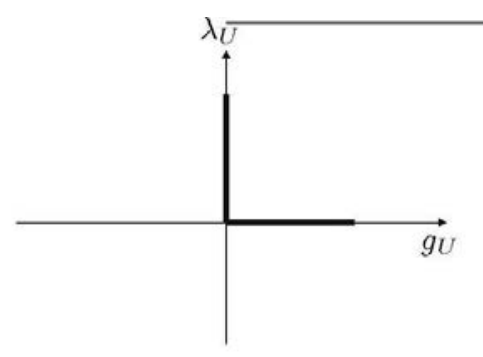

(b) Unilateral contact

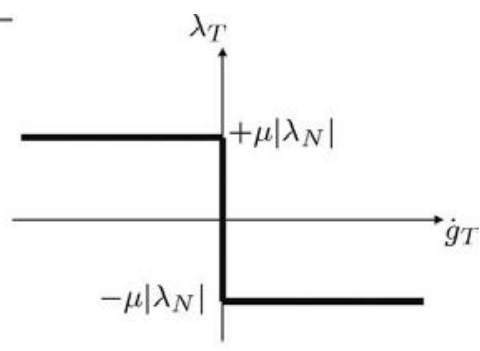

(c) Planar Coulomb friction

Fig. 2. Force laws for bilateral and unilateral contacts as well as planar CouLOMB friction.

Illustrations of implicit definitions of set-valued contact laws which fit into (15) and (16) are given in Remark 1.2. For more details, we refer to [10,2].

Remark 1.2 ((Set-valued contact laws)). Set-valued contact relations may be bilateral, unilateral or may describe a dry friction behaviour (cf. Fig. 2). With a sufficiently smooth local gap function $g_{B}(q, t) \in \mathbb{R}$, a bilateral contact (or a bilateral constraint, or a perfect ideal joint) $g_{B}(q, t)=0$ enforces physically a joint. The global contact force $r=W(q, t) \lambda_{B}$ is just a transformation of the local contact force $\lambda_{B}$ with $W(q, t)=\nabla_{q} g_{B}(q, t)$. Altogether, the set $\mathcal{N}_{C}$ in (15) can be defined as

$$
\mathcal{N}_{C}(q, v, r, t)=\left\{\begin{array}{l}
g_{B}(q, t)=0, \lambda_{B} \in \mathbb{R}, \\
r=W(q, t) \lambda_{B}, \\
\dot{g}_{B}(q, v, t)=W^{T}(q, t) v+\partial_{t} g_{B}(q, t)=0
\end{array}\right\} .
$$

Similarly, a unilateral contact with local gap function $g_{U}(q, t) \in \mathbb{R}$ is represented by Signorini-Fichera-conditions

$$
0 \leq g_{U} \perp \lambda_{U} \geq 0
$$

The symbol $\perp$ implies complementarity, i.e. $g_{U} \lambda_{U}=0$. The set $\mathcal{N}_{C}$ in (15) can be defined as

$$
\mathcal{N}_{C}(q, v, r, t)=\left\{\begin{array}{l}
0 \leq g_{U}(q, t) \perp \lambda_{U} \geq 0, \\
r=W(q, t) \lambda_{U}, \\
\dot{g}_{U}^{+}(q, v, t)=W^{T}(q, t) v^{+}+\partial_{t} g_{U}(q, t) \geq 0
\end{array}\right\} .
$$

The last equation in the definition of $\mathcal{N}_{C}$ in (19) implies that the velocity has to jump if $W^{T}(q, t) v^{-}+\partial_{t} g_{U}(q, t)<0$. This results in the introduction of an impact law. For instance, the Newton impact law with a coefficient of restitution $\epsilon_{N} \in[0,1]$ yields the following definition of the set $\mathcal{N}_{I}$

$$
\mathcal{N}_{I}\left(q_{j}, t_{j}, p_{j}, v_{j}\right)=\left\{\begin{array}{l}
0 \leq g_{U}^{+}\left(q_{j}, t_{j}\right)+\epsilon_{N} g_{U}^{-}\left(q_{j}, t_{j}\right) \perp \Lambda_{U} \geq 0, \\
p_{j}=W\left(q_{j}, t_{j}\right) \Lambda_{U}, \\
\dot{g}_{U}^{ \pm}\left(q_{j}, v_{j}, t_{j}\right)=W^{T}\left(q_{j}, t_{j}\right) v_{j}^{ \pm}+\partial_{t} g_{U}\left(q_{j}, t_{j}\right) \geq 0
\end{array}\right\} .
$$

For establishing Coulomb's law of dry friction, local contact forces are split in a component $\lambda_{N}$ normal to the contact tangent plane and in a tangential component $\lambda_{T}$ in the tangent plane. In non-degenerate cases, Coulomb's friction law is given as follows:

$$
\begin{aligned}
& \dot{g}_{T}=0 \Rightarrow\left\|\lambda_{T}\right\| \leq \mu\left|\lambda_{N}\right|, \\
& \dot{g}_{T} \neq 0 \quad \Rightarrow \lambda_{T}=-\frac{\dot{g}_{T}}{\left\|\dot{g}_{T}\right\|} \mu\left|\lambda_{N}\right|
\end{aligned}
$$

where $\mu>0$ is the coefficient of friction and $\dot{g}_{T}(q, v, t)$ is the local tangent velocity. The set $\mathcal{N}_{C}$ can similarly be defined as in (19). 
Interactions may be the root of impulsive behaviour. When a unilateral contact relation closes at time $t_{j}$, it has to be evaluated as impact relation to ensure the validity of the constraints after impact time. At this moment, all closed set-valued contact relations are influenced. Hence, also the bilateral and frictional relations have to be considered as impact relations.

Problem 1.1 is a measure differential inclusion (MDI). It uses a weak description of time derivatives in terms of measures. As in the modern theory of partial differential equations (PDE), Problem 1.1 can directly be interpreted in the sense of distributions. We will see that this concept is even more general and that it offers the connection to Galerkin schemes known from the numerical treatment of PDEs. To this end, one achieves the following problem.

Problem 1.3 ((Distribution differential inclusion)). Solve

$$
\begin{aligned}
& \left\langle\dot{q}, \varphi_{q}\right\rangle=\left\langle v, \varphi_{q}\right\rangle, \quad \forall \varphi_{q} \in \mathcal{D}(I), \\
& \left\langle D v, \varphi_{v}\right\rangle=\left\langle m^{-1} f, \varphi_{v}\right\rangle+\left\langle m^{-1} \mathrm{~d} i, \varphi_{v}\right\rangle, \quad \forall \varphi_{v} \in \mathcal{D}(I)
\end{aligned}
$$

together with the initial conditions (4) and (5).

We need some explanations, which also deepen the relation between (4)-(8), Problem 1.1 and Problem 1.3. The measure Eq. (9) is defined by

$$
\int \dot{q} \varphi_{q} \mathrm{~d} t=\int v \varphi_{q} \mathrm{~d} t
$$

being valid for all $\varphi_{q}$ such that the integrals make sense. In particular, Eq. (24) holds for all $\varphi_{q} \in \mathcal{D}(I)$. Hence, $\dot{q}$ (and respectively $v$ ) can be identified with its distribution $T_{\dot{q}}$ (resp. $T_{v}$ ) or with the linear functional $\langle\dot{q}, \cdot\rangle=\int \dot{q} \cdot \mathrm{d} t$ (resp. $\left.\langle v, \cdot\rangle=\int v \cdot \mathrm{d} t\right)$. One interprets the constructors $\dot{q}$ and $v$ as elements of $\mathcal{D}^{*}(I)$ and writes (22) instead of (24).

Whereas for $q$ there exists a weak time derivative $\dot{q}$, the derivative $D v$ of $v$ exists at least in a distributional sense. If $v$ were absolutely continuous, the distributional derivative definition (3) would exactly characterise the integration by parts formula

$$
\int \dot{v} \varphi_{v} \mathrm{~d} t:=-\int v \dot{\varphi}_{v} \mathrm{~d} t, \quad \forall \varphi_{v} \in \mathcal{D}(I)
$$

Thereby, we have to take our interpretation of absolutely continuous functions as elements of $\mathcal{D}^{*}(I)$ into consideration. Whereas a distributional derivative always exists, additional smoothness properties have to be checked afterwards. Then, a distributional derivative might be e.g. a weak derivative for absolutely continuous functions interpreted as elements of $\mathcal{D}^{*}(I)$ or even a classical derivative of differentiable functions interpreted as elements of $\mathcal{D}^{*}(I)$. In fact, the distributional derivative $D v$ of $v$, which is assumed to be a LBV function, is the differential measure $\mathrm{d} v$ and accordingly it has specific 'smoothness' properties, which are obviously not as strong as those of $\dot{q}$. To enforce the notation of derivatives, we continue using the more general description $D v$ instead of $\mathrm{d} v$. Finally in Problem 1.3, the involved distributions are on the one hand constructed by locally integrable functions $L_{\text {loc }}^{1}(I)$ and on the other hand by measures $\mathcal{M}(I)$ in the way which we already described in the Notation (see (2)).

In this sense, $L_{\text {loc }}^{1}(I)$ and $\mathcal{M}(I)$ can be identified with subspaces of $\mathcal{D}^{*}(I)$. Actually, elements of these subspaces map functions to $\mathbb{R}$ which are not elements of $\mathcal{D}(I)$. The test functions $\varphi_{q}$ and $\varphi_{v}$ do not have to be elements of $\mathcal{D}(I)$ or even of $\mathcal{C}^{\infty}$; only the occurring linear functionals have to be consistently defined for a suitable smoothness of $\varphi_{q}$ and $\varphi_{v}$. For the position, the test functions $\varphi_{q}$ do not need to be continuous because each element of $L_{\mathrm{loc}}^{1}(I)$ naturally defines a measure as a density function with respect to the Lebesgue measure. Finite evaluations of the primal-dual pairings are not a problem in practice for position test functions. For the velocity, at least function evaluations of $\varphi_{v}$ are necessary such that elements of $\mathcal{M}(I)$ can be consistently applied:

$$
\sum_{j}\left[\left[v_{j}\right]\right] \varphi_{v}\left(t_{j}\right)=\sum_{j} m_{j}^{-1} p_{j} \varphi_{v}\left(t_{j}\right)
$$

Hence, the test functions for the velocity $\varphi_{v}$ must be continuous at the impact times. 


\subsection{Integration methods}

Timestepping schemes next to event-driven schemes are well-known possibilities to integrate nonsmooth dynamical systems [2].

\subsubsection{Classical timestepping schemes}

Classical timestepping schemes, also called event-capturing schemes, discretize the equations of motion in Problem 1.1 including the constraints (15), (16) with integration order one and without resorting to an accurate event detection procedure. As the time step-size is never adapted, a large number of constraint transitions can be handled with increased computational efficiency when the influence of particular events is not as important as the mean.

Algorithm 1.4. Classic Moreau-Jean timestepping scheme [20,13]

INPUT time interval partition $\mathcal{I}$, inverse mass $m^{-1}$, external forces $f$, impact set $\mathcal{N}_{I}$, initial position $q_{0}$, initial velocity $v_{0}$, parameter $\theta$

$i \leftarrow 1$ INITIALIZE LOOP VARIABLE

WHILE $i \leq N$

- solve

$$
\left\{\begin{array}{l}
q_{i}=q_{i-1}+\Delta t_{i}\left[(1-\theta) v_{i-1}+\theta v_{i}\right] \\
v_{i}=v_{i-1}+\Delta t_{i}\left[(1-\theta) m_{i-1}^{-1} f_{i-1}+\theta m_{i}^{-1} f_{i}\right]+m_{i}^{-1} \Delta i_{i} \\
\text { with }\left(q_{i}, v_{i}, \Delta i_{i}, t_{i}\right) \in \mathcal{N}_{I}
\end{array}\right\}
$$

- $i \leftarrow i+1$

Algorithm 1.4 is a representative timestepping scheme. It defines numerical approximations $q_{i} \approx q\left(t_{i}\right), v_{i} \approx v\left(t_{i}\right)$, $m_{i}^{-1} \approx m^{-1}\left(q_{i}\right), f_{i} \approx f\left(t_{i}, q_{i}, v_{i}\right), \Delta i_{i} \approx \mathrm{d} i\left(\left[t_{i-1}, t_{i}\right)\right)$ and does not distinguish between contacts and impacts (cf. Remark 2.4) being evaluated on velocity level. A question of ongoing research is the solution of the nonlinear expressions forming the kernel of the algorithm. Note that another class of classical timestepping schemes exists which will not be discussed here. Details on these developments can be found in $[22,23,21]$

\subsubsection{Event-driven schemes}

Event-driven schemes, also known as event-tracking schemes, resolve the exact constraint transition times of Problem 1.1. Between the events, the motion of the system is computed by a classical integration method for differential algebraic equations (DAE). This is very accurate but the detection of events can be time consuming and is not possible for Zeno phenomena: the schemes become inconsistent. Moreover, event-driven schemes require the definition of small threshold parameters which depend strongly on the problem formulation. In practise, these thresholds are very difficult to tune in a robust way. Though if an underlying mathematical model exhibits only sparse events (large density of events or even finite accumulations of events are forbidden), event-driven schemes are most of the time our methods of choice.

\subsubsection{Higher order timestepping approaches}

We can find two different approaches for achieving consistent higher order timestepping schemes in the literature, which can also deal with finite accumulation of impacts, or large density of impacts with respect to the time-scale of study: augmented timestepping schemes and mixed timestepping schemes.

Augmented timestepping schemes [27,12]. Augmented timestepping schemes are extensions of classical timestepping schemes, e.g. of Moreau-Jean type $[20,13]$. If there is no velocity jump during an integration step, one uses classical augmentation strategies [7,11]: 
- Extrapolation techniques emanating from the basic classical timestepping scheme increase the integration order.

- Time step-size adaptation according to Richardson or using embedding methods permit automatic time step-size changes.

Often, the order extrapolation leads to instabilities with closed unilateral constraints because of chattering in the classical Aitken-Neville scheme or because of missing splitting between non-impulsive and impulsive force propagation. Further, order extrapolation cannot conceptually be scheduled in parallel. These items have led to the utilisation of methods with fixed integration order even in the non-impulsive phases. However, the main problem is to find a consistent treatment of impulsive episodes. This is usually done by heuristics: one uses the classical timestepping scheme and one has to decide about time step-size adaptation.

Mixed timestepping schemes [1,8]. Mixed timestepping schemes combine DAE methods for non-impulsive episodes with classical timestepping schemes for impulsive phases without resolving the exact constraint transition times. They benefit from the classical theory in non-impulsive segments exactly as augmented timestepping schemes. They are also seriously affected by appropriate time step-size adaptation for impulsive episodes.

Step-size adaptation. Both augmented and mixed procedures suffer mainly from lacking appropriate time step-size adaptation strategies in impulsive periods. Usually, one starts from the classical approach based on control theory $[7,11]$ and uses additional heuristics respecting the idea behind timestepping schemes:

- Anticipating gap-estimations [12,8] or retrospective time step bisection [27], [1, for mixed timestepping] ensure sufficiently exact detection of possible velocity jumps.

- Time step-size switching $\Delta t_{\text {impulsive }}=\mathcal{O}\left(\Delta t_{\text {smooth }}^{p+1}\right)$ couples non-impulsive and impulsive regions using the integration order $p$ of the non-impulsive propagation [27], [1, for mixed timestepping].

- Error estimation is based on not adapted [12] or adapted [1, for classical timestepping] Richardson strategies with some additional heuristics, i.e.

- exclusion of the possibly jumping velocities in the error estimation [12], [1, for classical timestepping],

- discussion of appropriate norms [1, for classical timestepping],

- preferable interval-by-interval separation of possible velocity jumps [12,1],

- dependence on penetration for closed contacts [1, for classical timestepping].

Mainly because of missing smoothness, it is very difficult to derive an appropriate time step-size adaptation respecting the tolerance demands. All mentioned items, i.e. event prediction, norm selection, error estimation and time step-size selection, have not been solved satisfactory for impulsive transitions yet. We will have to struggle with exactly the same setting when we prepare timestepping schemes based on time discontinuous Galerkin methods to industrial problems regarding efficiency.

\section{Time discontinuous Galerkin methods}

To consistently improve the behaviour during smooth episodes, we embed the classical timestepping schemes in time discontinuous Galerkin (TDG) methods. Our article follows [15], which is considered to be the first contribution. Also [14,9,5,3] have motivated our approach.

We start from Problem 1.3 and would like to define proper test functions and a finite dimensional basis for the discrete solution. We assume:

- test functions might have jumps across the intervals,

- test functions are continuous inside the intervals.

The first assumption leads to the expression of discontinuous Galerkin methods. The second claim states that there is not an instantaneous influence of the analytic nonsmooth dynamics on the numerical solution in-between an interval: the exact time of discontinuity is not resolved. 


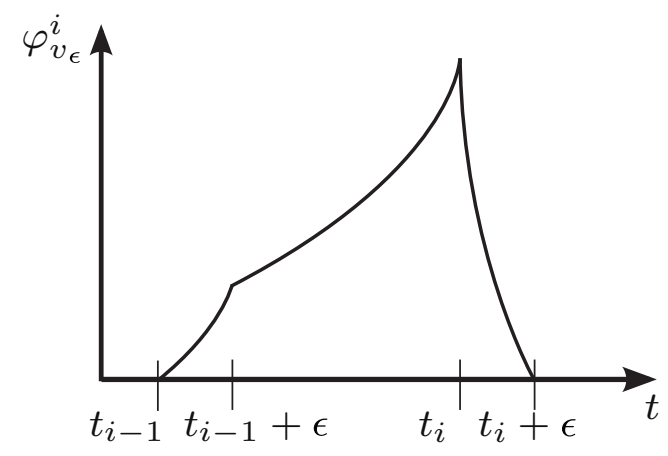

Fig. 3. Characteristic mollifier.

\subsection{Evaluations with discontinuous test functions}

It is not clear how to reinterpret (26) if we are using discontinuous test functions and their discontinuities coincide with those of the function of bounded variations $v$. Depending on the usage of appropriate mollifiers, i.e. smooth cutoff functions, we define the distributional derivative of a functional $v \in \mathcal{C}^{1}(\mathcal{I})$ applied to discontinuous functions $\varphi_{v} \in \mathcal{C}^{1}(\mathcal{I})[3]$. Let $\epsilon>0$ and $i$ an arbitrary index. E.g. with

$$
\chi_{\epsilon}^{i-}: \mathbb{R} \rightarrow \mathbb{R}, \quad t \mapsto \chi_{\epsilon}^{i-}(t):=\left\{\begin{array}{ll}
\left(t-t_{i-1}\right) / \epsilon & \text { for } t_{i-1} \leq t<t_{i-1}+\epsilon \\
1 & \text { for } t_{i-1}+\epsilon \leq t<t_{i} \\
1+\left(t_{i}-t\right) / \epsilon & \text { for } t_{i} \leq t<t_{i}+\epsilon \\
0 & \text { elsewhere }
\end{array},\right.
$$

we gain an absolutely continuous characteristic mollifier (cf. Fig. 3)

$$
\varphi_{v_{\epsilon}}^{i-}: \mathbb{R} \rightarrow \mathbb{R}, \quad t \mapsto \varphi_{v_{\epsilon}}^{i-}(t):=\varphi_{v}(t) \chi_{\epsilon}^{i-}(t)
$$

of $\varphi_{v}$ with support in $\left(t_{i-1}, t_{i}+\epsilon\right)$. The integration by parts formula yields

$$
\begin{aligned}
\int D^{-} v \varphi_{v_{\epsilon}}^{i-} \mathrm{d} t: & =-\int_{t_{i-1}}^{t_{i-1}+\epsilon} v \dot{\varphi}_{v_{\epsilon}}^{i-} \mathrm{d} t-\int_{t_{i-1}+\epsilon}^{t_{i}} v \dot{\varphi}_{v_{\epsilon}}^{i-} \mathrm{d} t-\int_{t_{i}}^{t_{i}+\epsilon} v \dot{\varphi}_{v_{\epsilon}}^{i-} \mathrm{d} t \\
& =\left[\left[v_{i}\right]\right] \varphi_{v_{\epsilon}}^{i-}\left(t_{i}\right)+\int_{t_{i-1}}^{t_{i}} \dot{v} \varphi_{v_{\epsilon}}^{i-} \mathrm{d} t+\int_{t_{i}}^{t_{i}+\epsilon} \dot{v} \varphi_{v_{\epsilon}}^{i-} \mathrm{d} t
\end{aligned}
$$

because of the continuity of $\varphi_{v_{\epsilon}}^{i-}$ in $t_{i-1}+\epsilon$ and $t_{i}$. In the limit $\epsilon \rightarrow 0$, we use $\chi_{\epsilon}^{i-}\left(t_{i}\right)=1$ and the theorem of Lebesgue to achieve

$$
\lim _{\epsilon \rightarrow 0} \int D^{-} v \varphi_{v_{\epsilon}}^{i-} \mathrm{d} t=\left[\left[v_{i}\right]\right] \varphi_{v}\left(t_{i}^{-}\right)+\int_{t_{i-1}}^{t_{i}} \dot{v} \varphi_{v} \mathrm{~d} t .
$$

Hence, we define with a partition of unity ansatz

$$
\left\langle D^{-} v, \varphi_{v}\right\rangle:=\sum_{i}\left[\left[v_{i}\right]\right] \varphi_{v}\left(t_{i}^{-}\right)+\sum_{i} \int_{t_{i-1}}^{t_{i}} \dot{v} \varphi_{v} \mathrm{~d} t .
$$

This expression focuses on discontinuities at the right border $t_{i}$ of $I_{i}$. Alternatively incorporating the left border $t_{i-1}$ of $I_{i}$ with a similar mollifier $\chi_{\epsilon}^{i+}$,

$$
\left\langle D^{+} v, \varphi_{v}\right\rangle:=\sum_{\dot{1}}\left[\left[v_{i-1}\right]\right] \varphi_{v}\left(t_{i-1}^{+}\right)+\sum_{i} \int_{t_{i-1}}^{t_{i}} \dot{v} \varphi_{v} \mathrm{~d} t
$$

is also a consistent definition. The discontindity evaluations also could have been totally omitted, which is physically not satisfactory. Further, both the left and the right border of $I_{i}$ could have been considered. This would result in two 
term recursions, i.e. multi-step methods, because of the three intervals $I_{i-1}, I_{i}$ and $I_{i+1}$ play a role in. The choice of the mollifier is crucial and might change both physics and numerical behaviour.

We use the expression $\mathrm{d} i^{ \pm}$instead of $\mathrm{d} i$ for the interaction measure.

\subsection{Timestepping schemes based on time discontinuous Galerkin methods}

We demonstrate the numerical approximation of Problem 1.1 with time discontinuous Galerkin methods and discuss its properties.

\subsubsection{Definition of the Galerkin approximation}

Let $\Phi_{q}^{h}, \Phi_{v}^{h} \subset \mathcal{C}^{0}(\mathcal{I})$ be finite dimensional subspaces for test functions with respective bases $\mathcal{B}_{\Phi_{q}^{h}}:=\left\{\varphi_{q_{k}}^{h}\right\}_{k}$ and $\mathcal{B}_{\Phi_{v}^{h}}:=\left\{\varphi_{v_{k}}^{h}\right\}_{k}$. Let further $\Psi_{\dot{q}}^{h}, \Psi_{v}^{h} \subset \mathcal{L B} \mathcal{V}(I)$ be conforming subspaces for the choice of $\dot{q}$ - and $v$-ansatz functions. The corresponding bases are given by $\mathcal{B}_{\Psi_{\dot{q}}^{h}}:=\left\{\psi_{\dot{q}_{k}}^{h}\right\}_{k}$ and $\mathcal{B}_{\Psi_{v}^{h}}:=\left\{\psi_{v_{k}}^{h}\right\}_{k}$. Then,

$$
\begin{aligned}
q^{h}: I \rightarrow \mathbb{R}, \quad t \mapsto q^{h}(t):=q_{0}+\sum_{k} \int_{t_{0}}^{t} \psi_{\dot{q}_{k}}^{h} \mathrm{~d} s \dot{q}_{k}^{h}, \\
v^{h}: I \rightarrow \mathbb{R}, \quad t \mapsto v^{h}(t):=\sum_{k} \psi_{v_{k}}^{h}(t) v_{k}^{h}
\end{aligned}
$$

is a representation of the numerical solution. The weights $\left\{\dot{q}_{k}^{h}\right\}_{k}$ and $\left\{v_{k}^{h}\right\}_{k}$ are specified later. Inserting these expressions into Problem 1.3 yields the discrete problem.

Problem 2.1 ((Petrov-Galerkin distribution differential inclusion)). Solve

$$
\begin{aligned}
& \sum_{k}\left\langle\psi_{\dot{q}_{k}}^{h}, \varphi_{q_{l}}^{h}\right\rangle \dot{q}_{k}^{h}=\sum_{k}\left\langle\psi_{v_{k}}^{h}, \varphi_{q_{l}}^{h}\right\rangle v_{k}^{h}, \quad \forall \varphi_{q_{l}}^{h} \in \Phi_{q}^{h}, \\
& \sum_{k}\left\langle D^{ \pm} \psi_{v_{k}}^{h}, \varphi_{v_{l}}^{h}\right\rangle v_{k}^{h}=\left\langle m^{-1} f, \varphi_{v_{l}}^{h}\right\rangle+\left\langle m^{-1} \mathrm{~d} i^{ \pm}, \varphi_{v_{l}}^{h}\right\rangle, \quad \forall \varphi_{v_{l}}^{h} \in \Phi_{v}^{h}
\end{aligned}
$$

together with the discrete initial conditions

$$
\begin{gathered}
q^{h}(0):=q_{0} \in \mathbb{R}, \\
v^{h}(0):=v_{0} \in \mathbb{R} .
\end{gathered}
$$

It is clear that $m^{-1}, f$, and $\mathrm{d} i^{ \pm}$are evaluated using $q^{h}$ and $v^{h}$. Contact and impact laws are evaluated to compute $\mathrm{d} i^{ \pm}$.

\subsubsection{Comparison with the classical Moreau-Jean timestepping scheme}

Problem 2.1 is a general description which does not give appropriate time discretization schemes in all cases. The quality of the schemes is highly depending on the ansatz and test function subspaces. What are primary drivers for their selection?

- Problem 1.1 depends on an initial value and describes a time-evolutionary solution. Also Problem 2.1 should state an evolution process not depending on future information at each point in time.

- Experience has shown that for the description of nonsmooth dynamical systems, one-step methods are more appropriate than multi-step methods due to the lack of regularities of the right hand side [2].

- For efficient evaluation of the primal-dual pairings, easy test and ansatz functions should be used. They have to represent the smoothness of the analytical problem depending on e.g. external forces but also on constraints. 

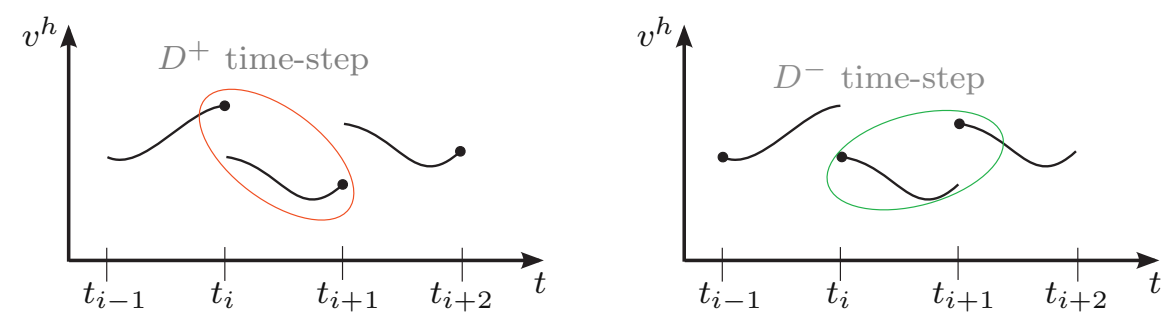

Fig. 4. Velocity jump interpretation for $D^{+}$and $D^{-}$.

One possibility to achieve these goals is the selection of piecewise polynomials. Choosing piecewise constant spaces $\Phi_{q}^{h}=\Phi_{v}^{h}=\Psi_{\dot{q}}^{h}=\Psi_{v}^{h}:=\mathcal{P}^{0}(\mathcal{I})$, characteristic functions generate canonical bases $\mathcal{B}_{\Phi_{q}^{h}}=\mathcal{B}_{\Phi_{v}^{h}}=\mathcal{B}_{\Psi_{\dot{q}}^{h}}=\mathcal{B}_{\Psi_{v}^{h}}:=\left\{\chi^{i}\right\}_{i}$. Focusing on the time interval $I_{i} \in \mathcal{I}$, we gain well-known classical timestepping schemes as a special case of Problem 2.1. We distinguish the alternative evaluations, i.e. $D^{ \pm}$and $\mathrm{d} i^{ \pm}$.

$D^{+}$and $\mathrm{d} i^{-}$.

Problem 2.2 ((Implicit Moreau-Jean timestepping scheme)). For $i \in \mathbb{N}$, solve

$$
\begin{aligned}
& q_{i}-q_{i-1}=v_{i} \Delta t_{i}, \\
& v_{i}-v_{i-1}=\int_{t_{i-1}}^{t_{i}} m^{-1} f \mathrm{~d} t+\left\langle m^{-1} \mathrm{~d} i^{-}, \chi^{i}\right\rangle
\end{aligned}
$$

together with the discrete initial conditions (37), (38).

Again, $m^{-1}, f$ and $\mathrm{d} i^{-}$are evaluated using $q^{h}$ and $v^{h}$. It is noteworthy that there has been some freedom.

- Due to the scheme in Problem 2.1, it is not stated how to select the weights $\left\{\dot{q}_{k}^{h}\right\}_{k}$ and $\left\{v_{k}^{h}\right\}_{k}$. We have chosen $\dot{q}_{k}^{h}$ and $v_{k}^{h}$ to coincide with the values of the numerical solution at the right end of the $k$ th interval (cf. Fig. 4 left panel). The constant velocity in $I_{i}$ is defined by $v_{i}=v^{h}\left(t_{i+1}^{-}\right)$and the velocity jump, due to $D^{+}$, occurs at the left side of $I_{i}$ where the impact, due to $\mathrm{d} i^{-}$, never occurs. The position propagation $q_{i}$ is derived from $\dot{q}_{i}$ by the fundamental theorem of calculus.

- The right hand side in (40) is not discretized. We have to choose appropriate quadrature rules which do not depend on discontinuities in the velocity, i.e. select an appropriate limit $v_{l}^{h \pm}$ if necessary, and avoid the resolution of impacts:

$$
\begin{aligned}
\int_{t_{i-1}}^{t_{i}} m^{-1} f \mathrm{~d} t & \approx\left(t_{i}-t_{i-1}\right) \sum_{l} \beta_{f_{l}} m^{-1}\left(q_{l}^{h}\right) f\left(t_{l}, q_{l}^{h}, v_{l}^{h \pm}\right), \\
\left\langle m^{-1} \mathrm{~d} i^{-}, \chi^{i}\right\rangle & \approx \sum_{l} m^{-1}\left(q_{l}^{h}\right) \Delta i_{l}
\end{aligned}
$$

with $\left(q_{l}^{h}, v_{l}^{h}, \Delta i_{l}, t_{l}\right) \in \mathcal{N}_{I}$ on velocity level. The classical Moreau-Jean timestepping scheme $(\theta=1)$ can be achieved with $\beta_{f_{1}}:=1, t_{1}:=t_{i}, q_{1}^{h}=q_{i}, v_{1}^{h}=v_{i}$ and $\left(q_{i}, v_{i}, \Delta i_{i}, t_{i}\right) \in \mathcal{N}_{I}$ on velocity level (cf. Algorithm 1.4).

$D^{-}$and $\mathrm{d} i^{-}$

Problem 2.3 ((Explicit Moreau-Jean timestepping scheme)). For $i \in \mathbb{N}$, solve

$$
\begin{aligned}
& q_{i}-q_{i-1}=v_{i-1} \Delta t_{i}, \\
& v_{i}-v_{i-1}=\int_{t_{i-1}}^{t_{i}} m^{-1} f \mathrm{~d} t+\left\langle m^{-1} \mathrm{~d} i^{-}, \chi^{i}\right\rangle
\end{aligned}
$$

together with the discrete initial conditions (37), (38). 
- We have chosen $\dot{q}_{k}^{h}$ and $v_{k}^{h}$ to coincide with the values of the numerical solutions at the left end of the $k$ th interval (cf. Fig. 4 right panel). The constant velocity in $I_{i}$ is defined by $v_{i-1}=v^{h}\left(t_{i}^{+}\right)$and the velocity jump, due to $D^{-}$, occurs at the right side together with the impact, due to $\mathrm{d} i^{-}$.

- The classical Moreau-Jean timestepping scheme with $\theta=0$ can be achieved with $\beta_{f_{1}}:=1, t_{1}:=t_{i-1}, q_{1}^{h}=q_{i-1}$, $v_{1}^{h}=v_{i-1}$ and $\left(q_{i}, v_{i}, \Delta i_{i}, t_{i}\right) \in \mathcal{N}_{I}$ on velocity level (cf. Algorithm 1.4).

$D^{+} / D^{-}$and $\mathrm{d} i^{+}$. With $\mathrm{d} i^{+}$, all impact evaluations take place in the semi-open interval $\left[t_{i-1}, t_{i}\right)$. The evaluation of the impact laws at the right border of $I_{i}$ is not maintained by the time discontinuous Galerkin scheme. Repeated tests have shown that this yields poor timestepping schemes [2]. We do not consider this case in the following.

Remark 2.4. Both Problem 2.2 and Problem 2.3 do not distinguish between contacts and impacts. The interaction measure $\mathrm{d} i^{-}$summarises both possibilities and is discretized directly. Hence, there is no splitting

$$
\Delta i_{l}=\left(t_{i}-t_{i-1}\right) \beta_{r_{l}} r\left(q_{l}^{h}\right)+p_{l}
$$

in non-impulsive and impulsive interactions and the direct application of higher order schemes would not be successful.

\section{Higher order timestepping}

For the development of higher order timestepping schemes based on time discontinuous Galerkin methods, we start from Problem 2.1. The procedure is similar to the embedding of the Moreau-Jean timestepping scheme in Section 2.2.2.

\subsection{Selection of bases functions}

The following questions arise when defining non-impulsive discrete position and velocity solutions inside an interval $I_{i}$. How can integrals with respect to arbitrary functions, e.g. $\left\langle m^{-1} f, \varphi_{v_{l}}^{h}\right\rangle$ or $\left\langle m^{-1} \mathrm{~d} i, \varphi_{v_{l}}^{h}\right\rangle$, be calculated efficiently? Is it possible to represent also the integrals with respect to polynomials of degree $2 M_{i}$, e.g. $\left\langle\psi_{\dot{q}_{k}}^{h}, \varphi_{q_{l}}^{h}\right\rangle$ or $\left\langle\psi_{v_{k}}^{h}, \varphi_{q_{l}}^{h}\right\rangle$, exactly by the same formula? This demand occurs when discretizing $I_{i}$ with $M_{i}+1$ points and nodal ansatz functions. The left and right border of $I_{i}$ play a special role according to Section 2. How can they be included as integration points? It turns out that the optimal quadrature rules of Gauß, Radau and Lobatto cannot positively respond to all our requirements. Including the borders of $I_{i}$ as integration points never allows exactness for polynomials of degree $2 M_{i}$. On the other side, Clenshaw-Curtis quadrature formulas have positive weights, can be evaluated fast and stable by Fast Fourier Transformation algorithms and are competitive for general integrands as well [28]. We choose the latter methods and mention that it is not a drawback that they are exact only for polynomials up to degree $M_{i}$. They evaluate the integrand at the Chebychev points $\left\{t_{i_{l}}\right\}_{l}$ for $M_{i} \neq 0$. For $M_{i}=0$, no rule exists but both $t_{i_{0}}=t_{i-1}$ and $t_{i_{0}}=t_{i}$ are popular choices. The weights with respect to $I_{i}$ and with respect to its lower sub-intervals satisfy

$$
\beta_{i_{l}}:=\beta_{f_{i_{l}}}=\beta_{r_{i_{l}}}=\frac{1}{\Delta t_{i}} \int_{I_{i}} l_{i_{l}} \mathrm{~d} t, \quad \beta_{i_{l}}\left(t^{*}\right):=\frac{1}{\Delta t_{i}} \int_{t_{i-1}}^{t^{*}} l_{i_{l}} \mathrm{~d} t
$$

with the classical pruned Lagrange polynomials

$$
l_{i_{l}}: I \rightarrow \mathbb{R}, \quad l_{i_{l}}(t):= \begin{cases}\prod_{j \neq l} \frac{t-t_{i_{j}}}{t_{i_{l}}-t_{i_{j}}}, & \text { for } t \in I_{i} \\ 0, & \text { for } t \notin I_{i}\end{cases}
$$

We use these pruned Lagrange polynomials to define piecewise polynomial nodal bases for test functions $\Phi_{q}^{h}=\Phi_{v}^{h}:=\mathcal{P}^{\alpha}(\mathcal{I})$ and for ansatz functions $\Psi_{\dot{q}}^{h}=\Psi_{v}^{h}:=\mathcal{P}^{\alpha}(\mathcal{I})$. This is a consistent approach, which actually yields a classical Galerkin scheme. Multi-index notation $\alpha:=\left(M_{1}, \ldots, M_{N}\right)$ allows for varying polynomial degrees for different 
elements of $\mathcal{I}$ if needed. Altogether, this results in respective $\left(N+\sum M_{i}\right)$-dimensional bases $\mathcal{B}_{\Phi_{q}^{h}}, \mathcal{B}_{\Phi_{v}^{h}}, \mathcal{B}_{\Psi_{\dot{q}}^{h}}$ and $\mathcal{B}_{\Psi_{v}^{h}}$. Their elements satisfy

$$
\varphi_{q_{k}}^{h}=\varphi_{v_{k}}^{h}=\psi_{\dot{q}_{k}}^{h}=\psi_{v_{k}}^{h}=l_{i_{l}} \text { with } k=\sum_{j=1}^{i-1}\left(M_{j}+1\right)+l .
$$

In the following, we can study easy evaluable one-step evolution processes just by focusing on one interval $I_{i}$ and by using the related index notation.

\subsection{Definition of the general scheme}

Stages are the values of position, velocity or acceleration approximations which coincide with the peaks of the nodal bases relative to a sub-interval $I_{i}$ :

$$
\begin{aligned}
& q_{i-1,0}^{h}=q_{i-1}^{h}, \quad q_{i-1,1}^{h}=q^{h}\left(t_{i_{1}}\right), \ldots, q_{i-1, M_{i}-1}^{h}=q^{h}\left(t_{i_{M_{i}-1}}\right), \quad q_{i-1, M_{i}}^{h}=q_{i}^{h}, \\
& \dot{q}_{i-1,0}^{h}=\dot{q}_{i-1}^{h+}, \quad \dot{q}_{i-1,1}^{h}=\dot{q}^{h}\left(t_{i_{1}}\right), \ldots, \dot{q}_{i-1, M_{i}-1}^{h}=\dot{q}^{h}\left(t_{i_{M_{i}-1}}\right), \quad \dot{q}_{i-1, M_{i}}^{h}=\dot{q}_{i}^{h-}, \\
& v_{i-1,0}^{h}=v_{i-1}^{h+}, \quad v_{i-1,1}^{h}=v^{h}\left(t_{i_{1}}\right), \ldots, v_{i-1, M_{i}-1}^{h}=v^{h}\left(t_{i_{M_{i}-1}}\right), \quad v_{i-1, M_{i}}^{h}=v_{i}^{h-}, \\
& \dot{v}_{i-1,0}^{h}=\dot{v}_{i-1}^{h+}, \quad \dot{v}_{i-1,1}^{h}=\dot{v}^{h}\left(t_{i_{1}}\right), \ldots, \dot{v}_{i-1, M_{i}-1}^{h}=\dot{v}^{h}\left(t_{i_{M_{i}-1}}\right), \quad \dot{v}_{i-1, M_{i}}^{h}=\dot{v}_{i}^{h-} .
\end{aligned}
$$

We insert the subspace specializations for one interval $I_{i}$, i.e. functions like in (48), in Problem 2.1 and we use $\mathrm{d} i^{-}$ because of stability reasons. On the one hand $\mathrm{d} i^{+}$evaluates impacts in the semi-open interval $\left[t_{i-1}, t_{i}\right)$ according to Section 2.2.2. On the other hand, Section 3.2.3 shows that $t_{i-1}$ will be the only reasonable candidate in this case. This is an explicit evaluation not ensuring the validity of the constraint after impact time; it is known to have bad properties for classical timestepping schemes [2].

Finally, we get the discrete initial conditions (37), (38). The position equation of Problem 2.1 yields equations

$$
\sum_{k} \int_{I_{i}} l_{i_{k}} l_{i_{l}} \mathrm{~d} t \dot{q}_{i-1, k}^{h}=\sum_{k} \int_{I_{i}} l_{i_{k}} l_{i_{l}} \mathrm{~d} t v_{i-1, k}^{h}
$$

for $l \in\left\{0, \ldots, M_{i}\right\}$. We have to distinguish if the velocity jump should occur at the left or right interval border (cf. Fig. 4). With (41), (42) and (45), we obtain the following formulations from the velocity equation of Problem 2.1.

\subsubsection{Velocity representation: $D^{+}$}

Search $v_{i-1, M_{i}}^{h}=v_{i}^{h-}$ knowing $v_{i-1}^{h-}$ with equations

$$
\left[v_{i-1,0}^{h}-v_{i-1}^{h-}\right] l_{i_{l}}\left(t_{i-1}^{+}\right)+\sum_{k} \int_{I_{i}} \dot{i}_{i_{k}} l_{i_{l}} \mathrm{~d} t v_{i-1, k}^{h}=\Delta t_{i} \sum_{k} \beta_{i_{k}} m_{i_{k}}^{-1}\left[f_{i_{k}}^{ \pm}+r_{i_{k}}\right] l_{i_{l}}\left(t_{i_{k}}^{ \pm}\right)+\sum_{k} m_{k}^{-1} p_{k} l_{i_{l}}\left(t_{i_{k}}^{-}\right)
$$

for $l \in\left\{0, \ldots, M_{i}\right\}$.

\subsubsection{Velocity representation: $D^{-}$}

Search $v_{i}^{h+}$ knowing $v_{i-1,0}^{h}=v_{i-1}^{h+}$ with equations

$$
\left[v_{i}^{h+}-v_{i-1, M_{i}}^{h}\right] l_{i_{l}}\left(t_{i}^{-}\right)+\sum_{k} \int_{I_{i}} \dot{l}_{i_{k}} l_{i_{l}} \mathrm{~d} t v_{i-1, k}^{h}=\Delta t_{i} \sum_{k} \beta_{i_{k}} m_{i_{k}}^{-1}\left[f_{i_{k}}^{ \pm}+r_{i_{k}}\right] l_{i_{l}}\left(t_{i_{k}}^{ \pm}\right)+\sum_{k} m_{k}^{-1} p_{k} l_{i_{l}}\left(t_{i_{k}}^{-}\right)
$$

for $l \in\left\{0, \ldots, M_{i}\right\}$. 


\subsubsection{Impact representation}

How should we choose the quadrature formula for the impacts in (54) and (55)? We assume that the discrete velocity behaviour inside an interval is continuously represented by the stage propagation. Hence, impacts are only allowed at the interval borders:

$$
\sum_{k} m_{k}^{-1} p_{k} l_{i_{l}}\left(t_{i_{k}}^{-}\right)=m_{i}^{-1} p_{i} l_{i_{l}}\left(t_{i}^{-}\right)
$$

with

$$
\left(q_{i}^{h}, v_{i}^{h \pm}, p_{i}, t_{i}\right) \in \mathcal{N}_{I}
$$

on velocity level. For $D^{+}$, we use $v_{i}^{h-}$, and for $D^{-}$, we use $v_{i}^{h+}$ (cf. Fig. 4). The discretization $p_{i}$ equals the right limit of the interaction impulse at $t_{i}$.

\subsubsection{Weighting integral representation: reduced evaluation [15]}

The order of the local error is governed by the evaluation of (41), (42) and (45) with quadrature rules. Without changing the order, we approximate the weighting integrals in (53), (54) and (55)

$$
\begin{aligned}
& \sum_{k} \int_{I_{i}} l_{i_{k}} l_{i_{l}} \mathrm{~d} t \dot{q}_{i-1, k}^{h} \stackrel{\mathrm{C}-\mathrm{C}}{\approx} \Delta t_{i} \sum_{k} \beta_{i_{k}} \dot{q}_{i-1, k}^{h} l_{i_{l}}\left(t_{i_{k}}^{ \pm}\right)=\Delta t_{i} \beta_{i_{l}} \dot{q}_{i-1, l}^{h}, \\
& \sum_{k} \int_{I_{i}} l_{i_{k}} l_{i_{l}} \mathrm{~d} t v_{i-1, k}^{h} \stackrel{\mathrm{C}-\mathrm{C}}{\approx} \Delta t_{i} \sum_{k} \beta_{i_{k}} v_{i-1, k}^{h} l_{i_{l}}\left(t_{i_{k}}^{ \pm}\right)=\Delta t_{i} \beta_{i_{l}} v_{i-1, l}^{h}, \\
& \sum_{k} \int_{I_{i}} \dot{l}_{i_{k}} l_{i_{l}} \mathrm{~d} t v_{i-1, k}^{h} \stackrel{\mathrm{C}-\mathrm{C}}{\approx} \Delta t_{i} \sum_{k} \beta_{i_{k}} \dot{v}_{i-1, k}^{h} l_{i_{l}}\left(t_{i_{k}}^{ \pm}\right)=\Delta t_{i} \beta_{i_{l}} \dot{v}_{i-1, l}^{h}
\end{aligned}
$$

by the same quadrature rule according to Clenshaw-Curtis (C-C) [28]. Thereby, we evaluate $l_{i_{l}}$ at the interior limit $t_{i_{k}}^{ \pm}$ of the sub-interval borders.

\subsubsection{Runge-Kutta representation}

Substitution of (58) and (59) into (53) yields the collocation of $M_{i}+1$ velocity stages

$$
\dot{q}_{i-1, l}^{h}=v_{i-1, l}^{h} .
$$

We will search position stages $\left\{q_{i-1, l}^{h}\right\}_{l}$ knowing $q_{i-1,0}^{h}$ with the fundamental theorem of calculus (cf. (67), (68), (72), (73)). The velocity expressions (54) and (55) are simplified to respective $M_{i}+1$ equations by evaluating the nodal bases and by inserting (60):

$$
\left.\begin{array}{ll}
D^{+}: & {\left[v_{i-1,0}^{h}-v_{i-1}^{h-}\right] l_{i_{l}}\left(t_{i-1}^{+}\right)} \\
D^{-}: & {\left[v_{i}^{h+}-v_{i-1, M_{i}}^{h}\right] l_{i_{l}}\left(t_{i}^{-}\right)}
\end{array}\right\}=\Delta t_{i} \beta_{i_{l}}\left\{m_{i_{l}}^{-1}\left[f_{i_{l}}^{ \pm}+r_{i_{l}}\right]-\dot{v}_{i-1, l}^{h}\right\}+m_{i}^{-1} p_{i} l_{i_{l}}\left(t_{i}^{-}\right) .
$$

For constant ansatz functions and appropriate definition of the integration point, e.g. either $t_{i}$ or $t_{i-1}$, Eq. (62) reduces to the implicit or explicit Moreau-Jean timestepping scheme. For at least linear ansatz functions, condition (62) expresses velocity jumps

$$
\begin{aligned}
& D^{+}: v_{i-1,0}^{h}=v_{i-1}^{h-}+\Delta t_{i} \beta_{i_{0}}\left\{m_{i_{0}}^{-1}\left[f_{i_{0}}^{+}+r_{i_{0}}\right]-\dot{v}_{i-1,0}^{h}\right\} \\
& D^{-}: v_{i}^{h+}=v_{i-1, M_{i}}^{h}+\Delta t_{i} \beta_{i_{M_{i}}}\left\{m_{i_{M_{i}}}^{-1}\left[f_{i_{M_{i}}}^{-}+r_{i_{M_{i}}}\right]-\dot{v}_{i-1, M_{i}}^{h}\right\}+m_{i}^{-1} p_{i}
\end{aligned}
$$

and respective $M_{i}$ stage relationships for accelerations in Table 1 . The values $\dot{v}_{i-1,0}^{h}$ or $\dot{v}_{i-1, M_{i}}^{h}$ are needed for the evaluation of (63) or (64) and are still missing. Fortunately, these are values of the acceleration, which is a polynomial 
Table 1

Stage relationship for accelerations.

\begin{tabular}{llc}
\hline Stage & $D^{+}$ & $D^{-}$ \\
\hline$l=0$ & $\dot{v}_{i-1,0}^{h}=?$ & $\dot{v}_{i-1,0}^{h}=m_{i_{0}}^{-1}\left[f_{i_{0}}^{+}+r_{i_{0}}\right]$ \\
$l \in\left\{1, \ldots, M_{i}-1\right\}$ & $\dot{v}_{i-1, M_{i}}^{h}=m_{i_{M_{i}}}^{-1}\left[f_{i_{M_{i}}}^{-}+r_{i_{M_{i}}}\right]+\frac{m_{i}^{-1} p_{i}}{\Delta t_{i} \beta_{M_{i}}}$ & $\dot{v}_{i-1}^{h}=m_{i_{l}}^{-1}\left[f_{i_{l}}+r_{i_{l}}\right]$ \\
$l=M_{i}$ & $\dot{v}_{i-1, M_{i}}^{h}=?$ \\
\hline
\end{tabular}

of degree $M_{i}-1$ in $I_{i}$. This polynomial can be uniquely represented by the known $M_{i}$ nodal values in Table 1 as well as by respective and appropriate pruned Lagrangian bases $\left\{\tilde{l}_{i_{k}}^{ \pm}\right\}_{k}[15]$ :

$$
D^{+}: \dot{v}^{h}(t)=\sum_{k=1}^{M_{i}} \tilde{l}_{i_{k}}^{+}(t) \dot{v}_{i-1, k}^{h}, \quad D^{-}: \dot{v}^{h}(t)=\sum_{k=0}^{M_{i}-1} \tilde{l}_{i_{k}}^{-}(t) \dot{v}_{i-1, k}^{h} .
$$

Now, we evaluate the acceleration polynomials at $t_{i-1}$ or $t_{i}$ and get $\dot{v}_{i-1,0}^{h}$ or $\dot{v}_{i-1, M_{i}}^{h}$. Eq. (65) will be also used to derive stage representations of the velocity with the fundamental theorem of calculus (cf. (70), (71), (74)). With

$$
\tilde{\beta}_{i_{l}}^{ \pm}:=\frac{1}{\Delta t_{i}} \int_{I_{i}} \tilde{l}_{i_{l}}^{ \pm} \mathrm{d} t, \quad \tilde{\beta}_{i_{l}}^{ \pm}\left(t^{*}\right):=\frac{1}{\Delta t_{i}} \int_{t_{i-1}}^{t^{*}} \tilde{l}_{i_{l}}^{ \pm} \mathrm{d} t,
$$

we obtain the following Runge-Kutta interpretation of higher order timestepping schemes based on time discontinuous Galerkin methods.

Problem 3.1 $\left(\left(D^{+}\right.\right.$timestepping scheme $\left.)\right)$. Let $M_{i}$ positive and $l \in\left\{0, \ldots, M_{i}\right\}$ for $i \in \mathbb{N}$. Solve simultaneously for the position

$$
\begin{aligned}
& q_{i-1, l}^{h}=q_{i-1}^{h}+\Delta t_{i} \sum_{k} \beta_{i_{k}}\left(t_{i_{l}}\right) v_{i-1, k}^{h}, \\
& q_{i}^{h}=q_{i-1}^{h}+\Delta t_{i} \sum_{k} \beta_{i_{k}} v_{i-1, k}^{h}
\end{aligned}
$$

and for the velocity

$$
\begin{aligned}
& v_{i-1,0}^{h}=v_{i-1}^{h-}+\Delta t_{i} \beta_{i_{0}}\left\{m_{i_{0}}^{-1}\left[f_{i_{0}}^{+}+r_{i_{0}}\right]-\sum_{k=1}^{M_{i}} \tilde{l}_{i_{k}}^{+}\left(t_{i-1}^{+}\right) m_{i_{k}}^{-1}\left[f_{i_{k}}^{-}+r_{i_{k}}\right]\right\}-\tilde{l}_{i_{M_{i}}}^{+}\left(t_{i-1}^{+}\right) \frac{\beta_{i_{0}}}{\beta_{i_{M_{i}}}} m_{i}^{-1} p_{i}, \\
& v_{i-1, l}^{h}=v_{i-1,0}^{h}+\Delta t_{i} \sum_{k=1}^{M_{i}} \tilde{\beta}_{i_{k}}^{+}\left(t_{i_{l}}\right) m_{i_{k}}^{-1}\left[f_{i_{k}}^{-}+r_{i_{k}}\right]+\tilde{\beta}_{i_{M_{i}}}^{+}\left(t_{i_{l}}\right) \frac{m_{i}^{-1} p_{i}}{\beta_{i_{M_{i}}}}, \\
& v_{i}^{h-}=v_{i-1,0}^{h}+\Delta t_{i} \sum_{k=1}^{M_{i}} \tilde{\beta}_{i_{k}}^{+} m_{i_{k}}^{-1}\left[f_{i_{k}}^{-}+r_{i_{k}}\right]+\tilde{\beta}_{i_{M_{i}}}^{+} \frac{m_{i}^{-1} p_{i}}{\beta_{i_{M_{i}}}}
\end{aligned}
$$

together with (37), (38) and $\left(q_{i-1, k}^{h}, v_{i-1, k}^{h}, r_{i_{k}}, t_{i_{k}}\right) \in \mathcal{N}_{C}$ on acceleration level as well as $\left(q_{i}^{h}, v_{i}^{h-}, p_{i}, t_{i}\right) \in \mathcal{N}_{I}$ on velocity level.

For $D^{-}$, the notation is easier as the jump information is not propagated along $I_{i}$.

Problem $3.2\left(\left(D^{-}\right.\right.$timestepping scheme $\left.)\right)$. Let $M_{i}$ positive and $l \in\left\{0, \ldots, M_{i}\right\}$ for $i \in \mathbb{N}$. Solve simultaneously for the position

$$
q_{i-1, l}^{h}=q_{i-1}^{h}+\Delta t_{i} \sum_{k} \beta_{i_{k}}\left(t_{i_{l}}\right) v_{i-1, k}^{h}
$$




$$
q_{i}^{h}=q_{i-1}^{h}+\Delta t_{i} \sum_{k} \beta_{i_{k}} v_{i-1, k}^{h}
$$

and for the velocity

$$
\begin{aligned}
v_{i-1, l}^{h}= & v_{i-1}^{h+}+\Delta t_{i} \sum_{k=0}^{M_{i}-1} \tilde{\beta}_{i_{k}}^{-}\left(t_{i_{l}}\right) m_{i_{k}}^{-1}\left[f_{i_{k}}^{+}+r_{i_{k}}\right], \\
v_{i}^{h+}= & v_{i-1, M_{i}}^{h}+\Delta t_{i} \beta_{i_{M_{i}}}\left\{m_{i_{M_{i}}}^{-1}\left[f_{i_{M_{i}}}^{-}+r_{i_{M_{i}}}\right]-\sum_{k=0}^{M_{i}-1} \tilde{l}_{i_{k}}^{-}\left(t_{i}^{-}\right) m_{i_{k}}^{-1}\left[f_{i_{k}}^{+}+r_{i_{k}}\right]\right\} \\
& +m_{i}^{-1} p_{i}
\end{aligned}
$$

together with (37), (38) and $\left(q_{i-1, k}^{h}, v_{i-1, k}^{h}, r_{i_{k}}, t_{i_{k}}\right) \in \mathcal{N}_{C}$ on acceleration level as well as $\left(q_{i}^{h}, v_{i}^{h+}, p_{i}, t_{i}\right) \in \mathcal{N}_{I}$ on velocity level.

\subsection{Trapezoidal rules}

For linear velocity discretizations, Problems 3.1 and 3.2 reduce to trapezoidal rules. Algorithm 3.3 is the implicit trapezoidal rule with an implicit retrospect for the first stage $v_{i-1,0}$ of the velocity. The method resembles the classical Moreau-Jean timestepping scheme for $\theta=1 / 2$, i.e. Algorithm 1.4, and the two stage Lobatto schemes, IIIA for position and IIIC for velocity [11]. Contacts are evaluated on acceleration level leading to a method for ordinary differential equations (ODE-method), impacts are calculated on velocity level. Algorithm 3.4 is the implicit trapezoidal rule with an explicit Euler forecast for the second stage $v_{i-1,1}$ of the velocity. The procedure is similar to the classical Moreau-Jean timestepping scheme for $\theta=1 / 2$, i.e. Algorithm 1.4, and to the two stage Lobatto schemes, IIIA for position and III for velocity [11]. Contacts are evaluated on acceleration level leading to an ODE-method, impacts are calculated on velocity level.

Algorithm 3.3. $D^{+}$linear timestepping scheme: 'contemplating' trapezoidal rule

INPUT time interval partition $\mathcal{I}$, inverse mass $m^{-1}$, external forces $f$, contact set $\mathcal{N}_{C}$, impact set $\mathcal{N}_{I}$, initial position $q_{0}$, initial velocity $v_{0}^{-}$

$i \leftarrow 1$ INITIALIZE LOOP VARIABLE

WHILE $i \leq N$

- solve

$$
\left\{\begin{aligned}
& q_{i-1,0}=q_{i-1} \\
& q_{i-1,1}=q_{i-1}+\frac{\Delta t_{i}}{2}\left\{v_{i-1,0}+v_{i-1,1}\right\} \\
& q_{i}=q_{i-1}+\frac{\Delta t_{i}}{2}\left\{v_{i-1,0}+v_{i-1,1}\right\} \\
& v_{i-1,0}=v_{i-1}^{-}+\frac{\Delta t_{i}}{2}\left\{m_{i-1}^{-1}\left[f_{i-1}^{+}+r_{i-1}^{+}\right]-m_{i}^{-1}\left[f_{i}^{-}+r_{i}^{-}\right]\right\}-m_{i}^{-1} p_{i} \\
& v_{i-1,1}=v_{i-1}^{-}+\frac{\Delta t_{i}}{2}\left\{m_{i-1}^{-1}\left[f_{i-1}^{+}+r_{i-1}^{+}\right]+m_{i}^{-1}\left[f_{i}^{-}+r_{i}^{-}\right]\right\}+m_{i}^{-1} p_{i} \\
& v_{i}^{-}=v_{i-1}^{-}+\frac{\Delta t_{i}}{2}\left\{m_{i-1}^{-1}\left[f_{i-1}^{+}+r_{i-1}^{+}\right]+m_{i}^{-1}\left[f_{i}^{-}+r_{i}^{-}\right]\right\}+m_{i}^{-1} p_{i} \\
&\left(q_{i-1,0}, v_{i-1,0}, r_{i-1}^{+}, t_{i-1}\right),\left(q_{i-1,1}, v_{i-1,1}, r_{i}^{-}, t_{i}\right) \in \mathcal{N}_{C},\left(q_{i}, v_{i}^{-}, p_{i}, t_{i}\right) \in \mathcal{N}_{I}
\end{aligned}\right\}
$$


Algorithm 3.4. $D^{-}$linear timestepping scheme: 'forecasting' trapezoidal rule

INPUT time interval partition $\mathcal{I}$, inverse mass $m^{-1}$, external forces $f$, contact set $\mathcal{N}_{C}$, impact set $\mathcal{N}_{I}$, initial position $q_{0}$, initial velocity $v_{0}^{+}$

$i \leftarrow 1$ INITIALIZE LOOP VARIABLE

WHILE $i \leq N$

- solve

$$
\left\{\begin{array}{l}
q_{i-1,0}=q_{i-1} \\
q_{i-1,1}=q_{i-1}+\frac{\Delta t_{i}}{2}\left\{v_{i-1,0}+v_{i-1,1}\right\} \\
q_{i}=q_{i-1}+\frac{\Delta t_{i}}{2}\left\{v_{i-1,0}+v_{i-1,1}\right\} \\
v_{i-1,0}=v_{i-1}^{+} \\
v_{i-1,1}=v_{i-1}^{+}+\Delta t_{i} m_{i-1}^{-1}\left[f_{i-1}^{+}+r_{i-1}^{+}\right] \\
v_{i}^{+}=v_{i-1}^{+}+\frac{\Delta t_{i}}{2}\left\{m_{i-1}^{-1}\left[f_{i-1}^{+}+r_{i-1}^{+}\right]+m_{i}^{-1}\left[f_{i}^{-}+r_{i}^{-}\right]\right\}+m_{i}^{-1} p_{i} \\
\left(q_{i-1,0}, v_{i-1,0}, r_{i-1}^{+}, t_{i-1}\right),\left(q_{i-1,1}, v_{i-1,1}, r_{i}^{-}, t_{i}\right) \in \mathcal{N}_{C},\left(q_{i}, v_{i}^{+}, p_{i}, t_{i}\right) \in \mathcal{N}_{I}
\end{array}\right\}
$$

- $i \leftarrow i+1$

\section{Experimental convergence analysis}

Because of (61) and Table $1, D^{+}$and $D^{-}$timestepping schemes are collocating ODE-methods inside each nonimpulsive interval $I_{i}$ [7]. Hence, the local error for non-impulsive episodes only depends on the adopted quadrature rule.

Theorem 4.1 ((Order of local error.)). Using Clenshaw-Curtis quadrature rules, the order of the local error for Problems 3.1 and 3.2 satisfies

$$
p=M_{i}+1
$$

in sufficiently smooth intervals $I_{i}$.

Proof. cf. [7, Theorem 6.40]

This is very good news: whenever we have a non-impulsive propagation of state and (contact) forces, the local error is automatically of higher order, i.e. the numerical approximation is improved for a consistent integration scheme in general. However, we do not know anything about errors due to velocity or interaction jumps. As they are globally propagated, the global error will be affected from them. But how? We analyse exemplary the bouncing ball in three different situations: free flight (Problem 4.2), rest phase (Problem 4.3) and a combination of free flight with finite accumulation of impacts (Problem 4.4) [1]. We will see that our examples support Theorem 4.1 and indicate an order drop due to jumping velocities or interactions.

The bouncing ball defines a decoupled example because there is only one interaction possibility. The analytical solution of our settings is never exactly represented by the numerical approximations. Algorithm 1.4 with $\theta=1$ proposes piecewise linear position, as well as piecewise constant velocity and interaction discretizations. With Algorithm 3.4, we have piecewise quadratic positions, piecewise linear velocities and piecewise quadratic interactions. In a Python ${ }^{1}$

\footnotetext{
1 http://www.python.org/.
} 


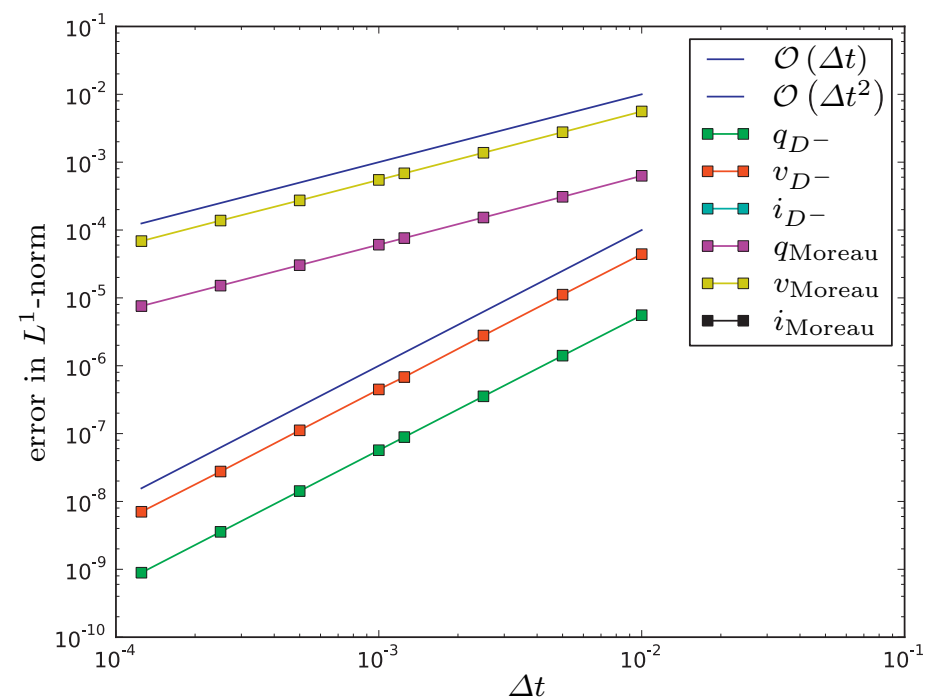

Fig. 5. Experimental convergence analysis: free flight.

implementation, we paid attention to evaluate the local and global error at least as exact as the timestepping discretizations. SciPy's ${ }^{2}$ barycentric interpolation for the velocities, as well as Hermite interpolation for positions and interactions are exact and efficient dense output formulas [11]. SciPy's Gauss-Konrod quadrature provides appropriate error formulas in $L^{1}$-norm for position, velocity and interactions.

Problem 4.2 ((Bouncing ball: free flight)). Discuss the scalar initial value problem

$$
\begin{aligned}
& q(0):=1, \quad v(0):=0, \\
& \dot{q}=v, \quad \dot{v}=-10 t^{2}
\end{aligned}
$$

in terms of measures.

The analytical solution is given by

$$
q(t)=1-\frac{5}{6} t^{4}, \quad v(t)=-\frac{10}{3} t^{3}, \quad i(t)=0 .
$$

During free flight, the state is of order two for Algorithm 3.4 and of order one for Algorithm 1.4 with $\theta=1$ (cf. Fig. 5). The interaction is zero and resolved exactly.

Problem 4.3 ((Bouncing ball: rest phase)). Discuss the scalar initial value problem

$$
\begin{aligned}
& q(0):=0, \quad v(0):=0, \\
& \dot{q}=v, \quad \dot{v}=-10 t^{2}+r, \\
& 0 \leq q \perp r \geq 0
\end{aligned}
$$

in terms of measures.

The analytical solution is given by

$$
q(t)=0, \quad v(t)=0, \quad i(t)=\frac{10}{3} t^{3} .
$$

During rest phase, the interaction is of order two for Algorithm 3.4 and of order one for Algorithm 1.4 with $\theta=1$ (cf. Fig. 6). The state is zero and resolved exactly.

\footnotetext{
2 http://www.scipy.org/.
} 


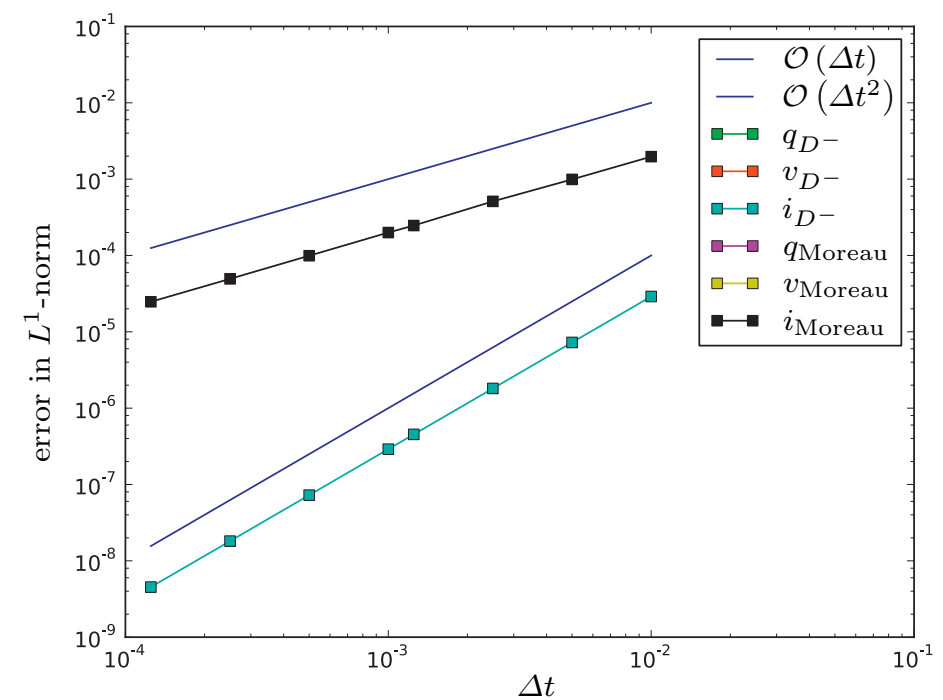

Fig. 6. Experimental convergence analysis: rest phase.

Problem 4.4 ((Bouncing ball: combined analysis)). Given the Newton restitution coefficient $\epsilon_{N}=0.5$, discuss the scalar initial value problem

$$
\begin{aligned}
& q(0):=1, \quad v(0):=0, \\
& \dot{q}=v, \quad \dot{v}=-2, \\
& v_{j}^{+}=v_{j}^{-}+\max \left\{0,-\left(1+\epsilon_{N}\right) v_{j}^{-}\right\} \text {if } q_{j}=0
\end{aligned}
$$

in terms of measures.

The analytical solution is given by

free flight $-0 \leq t<1$

$$
q(t)=1-t^{2}, \quad v(t)=-2 t, \quad i(t)=0
$$

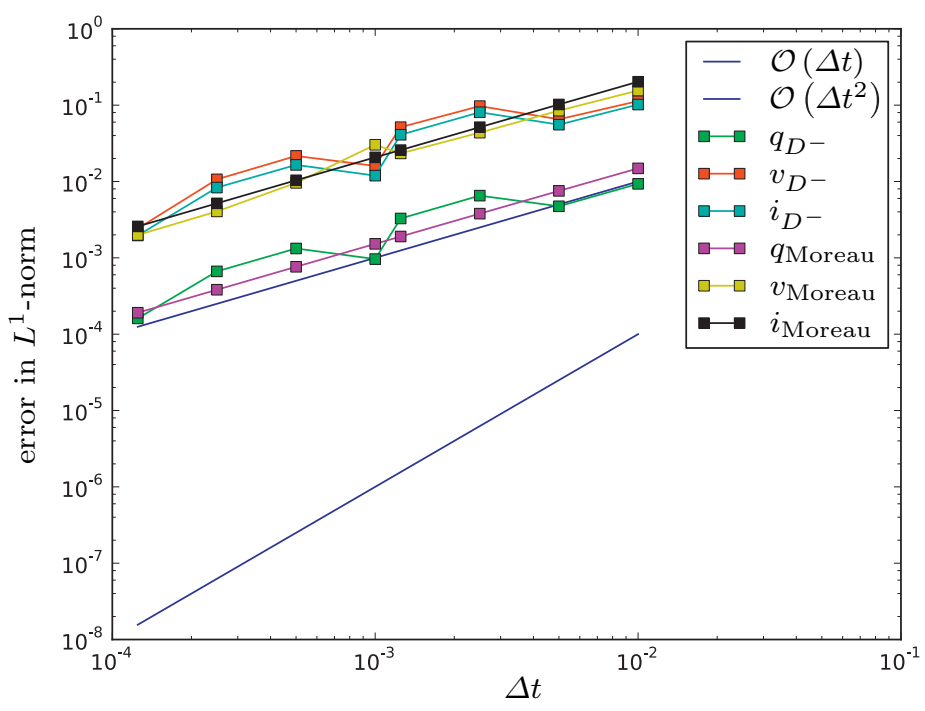

Fig. 7. Experimental convergence analysis: combined analysis. 


$$
\begin{aligned}
& \text { Zeno state }-\forall n \in \mathbb{N}_{0}: 3-\frac{1}{2^{n-1}} \leq t<3-\frac{1}{2^{n}} \\
& \begin{array}{c}
q(t)=-(t-3)^{2}-\frac{3}{2^{n}}(t-1)+\frac{1}{2^{n-1}}\left(3-\frac{1}{2^{n}}\right), \\
v(t)=-2(t-3)-\frac{3}{2^{n}}, \\
i(t)=\sum_{k=0}^{n} \frac{3}{2^{k}} .
\end{array}
\end{aligned}
$$

For the combined analysis, the global error of state and interaction is of order one for both Algorithm 3.4 and Algorithm 1.4 with $\theta=1$ (cf. Fig. 7).

\section{Conclusion}

In this paper, we have shortly summarised the state-of-the-art description of nonsmooth dynamical systems with either measure or distribution differential inclusions. Two classic integration methods have been identified for this type of evolution problems: event-driven and timestepping schemes. The intrinsic difficulty of event-driven integration is its high effort of event detection and inconsistency for Zeno phenomena. The drawback of classic timestepping schemes is their low integration order; recently, this had been tackled with augmentation and mixing to achieve both higher order in non-impulsive regions and the representation of infinitely many events. We have proposed a new strategy offering both a consistent embedding in time discontinuous Galerkin methods, as well as a splitting of nonimpulsive and impulsive force propagation. The framework has been developed in its full generality with mollifier functions, Clenshaw-Curtis quadrature rules and appropriate impact representation. Altogether, we have stated two Runge-Kutta collocation families as resulting timestepping methods. The order of the local error only depends on the order of the underlying quadrature rule for non-impulsive episodes. Choosing piecewise constant ansatz and test functions on velocity level, the classic explicit and implicit Moreau-Jean timestepping schemes have been found out to be special cases of the general method. For the piecewise linear case, the two families relate to a 'forecasting' and to a 'contemplating' trapezoidal rule. An experimental convergence analysis discusses the bouncing ball example in different episodes. We compare the properties of the constant ansatz to the characteristics of the linear ansatz. Whereas we have always integration order one for the Moreau-Jean timestepping, the new linear scheme offers integration order two in non-impulsive phases. This observation matches exactly the expectations and should be the starting point for further investigations. Can we derive any theoretical results about the global order of timestepping schemes? How does the proposed scheme perform for multi-dimensional systems with coupled multi-collisions, e.g. how can the contact and impact laws be evaluated separately? How can splitting methods improve timestepping schemes in these cases, e.g. using general DAE-methods instead of ODE-methods for non-impulsive episodes? What is necessary to define consistent automatic time step-size adaptations for timestepping schemes? Answers to these questions are important for even more successful time integration of nonsmooth industrial examples.

\section{Acknowledgements}

This work has been supported by the French National Research Agency (ANR) through COSINUS program (project SALADYN $^{3}$ ANR-08-COSI-014). The first author has been granted by an INRIA ${ }^{4}$ postdoctoral fellowship.

\section{References}

[1] V. Acary, Higher order event capturing time-stepping schemes for nonsmooth multibody systems with unilateral constraints and impacts, Applied Numerical Mathematics, 2012, http://dx.doi.org/10.1016/j.apnum.2012.06.026.

\footnotetext{
${ }^{3}$ http://saladyn.inria.gforge.fr/.

4 http://www.inria.fr/.
} 
[2] V. Acary, B. Brogliato, Numerical Methods for Nonsmooth Dynamical Systems: Applications in Mechanics and Electronics, Volume 35 of Lecture notes in applied and computational mechanics, 1st ed., Springer, Berlin, 2008.

[3] J. Alberty, C. Carstensen, Discontinuous Galerkin time discretization in elastoplasticity: motivation, numerical algorithms, and applications, Computer Methods in Applied Mechanics and Engineering 191 (2002) 4949-4968.

[4] P. Ballard, The dynamics of discrete mechanical systems with perfect unilateral constraints, Archive for Rational Mechanics and Analysis 154 (2000) 199-274.

[5] O. Bauchau, Computational schemes for flexible, nonlinear multi-body systems, Multibody System Dynamics 2 (1998) 169-225.

[6] B. Brogliato, T. ten Dam, L. Paoli, F. Genot, M. Abadie, Numerical simulation of finite dimensional multibody nonsmooth mechanical systems, Applied Mechanics Reviews 55 (2002) 107-150.

[7] P. Deuflhard, F. Bornemann, Scientific Computing with Ordinary Differential Equations, Volume 42 of Texts in Applied Mathematics, Springer, New York, 2002.

[8] B. Esefeld, H. Ulbrich, A hybrid integration scheme for nonsmooth mechanical systems, in: in: Multibody Dynamics 2011, Eccomas Thematic Conference, Brussels, 4th until 7th July, 2011.

[9] D. Estep, A posteriori error bounds and global error control for approximation of ordinary differential equations, SIAM Journal on Numerical Analysis 32 (1995) 1-48.

[10] C. Glocker, Set-valued Force Laws in Rigid Body Dynamics: Dynamics of Non-smooth Systems, Volume 1 of Lecture Notes in Applied and Computational Mechanics, 1st ed., Springer, Berlin, 2001.

[11] E. Hairer, G. Wanner, Solving Ordinary Differential Equations II: Stiff and Differential-Algebraic-Problems, Volume 14 of Springer Series in Computational Mathematics, 2nd rev. ed., 1st Softcover Printing, Springer, Berlin, 2010.

[12] R. Huber, H. Ulbrich, Higher order integration of non-smooth dynamical systems using parallel computed extrapolation methods based on time-stepping schemes, in: Proceedings of 1st Joint International Conference on Multibody System Dynamics, Lappeenranta, 25th-27th May, 2010.

[13] M. Jean, The nonsmooth contact dynamics method, Computer Methods in Applied Mechanics and Engineering 177 (1999) $235-257$.

[14] C. Johnson, Error estimates and adaptive time-step control for a class of one-step methods for stiff ordinary differential equations, SIAM Journal on Numerical Analysis 25 (1988) 908-926.

[15] P. Lasaint, P.A. Raviart, On a finite element method for solving the neutron transport equation, in: in: Symposium on Mathematical Aspects of Finite Elements in Partial Differential Equations, Madison, 1st until 3rd April, 1974.

[16] R.I. Leine, N. van de Wouw, Stability and Convergence of Mechanical Systems with Unilateral Constraints, Volume 36 of Lecture Notes in Applied and Computational Mechanics, Springer, Berlin, 2008.

[17] M. Monteiro Marques, Differential Inclusions in Nonsmooth Mechanical Problems. Shocks and Dry Friction, Progress in Nonlinear Differential Equations and their Applications, vol. 9, Birkhauser, Basel, 1993.

[18] J.J. Moreau, Bounded variation in time, in: Topics in Nonsmooth Mechanics, Birkhauser, Basel, 1988, pp. 1-74.

[19] J.J. Moreau, Unilateral contact and dry friction in finite freedom dynamics, in: Nonsmooth Mechanics and Applications, Springer, Wien, 1988, pp. $1-82$.

[20] J.J. Moreau, Numerical aspects of the sweeping process, Computer Methods in Applied Mechanics and Engineering 177 (1999) $329-349$.

[21] L. Paoli, An existence result for non-smooth vibro-impact problems, Journal of Differential Equations 211 (2005) $247-281$.

[22] L. Paoli, M. Schatzman, A numerical scheme for impact problems I: the one-dimensional case, SIAM Journal on Numerical Analysis 40 (2002) $702-733$.

[23] L. Paoli, M. Schatzman, A numerical scheme for impact problems II: the multi-dimensional case, SIAM Journal on Numerical Analysis 40 (2002) 734-768.

[24] F. Pfeiffer, Mechanical System Dynamics, Volume 40 of Lecture Notes in Applied and Computational Mechanics, corr. 2nd printing, Springer, Berlin, 2008.

[25] M. Schatzman, A class of nonlinear differential equations of second order in time, Nonlinear Analysis 2 (1978) 355-373.

[26] D. Stewart, Dynamics with Inequalities, SIAM, Philadephia, 2011.

[27] C. Studer, Numerics of Unilateral Contacts and Friction: Modeling and Numerical Time Integration in Non-smooth Dynamics, Volume 47 of Lecture Notes in Applied and Computational Mechanics, Springer, Berlin, 2009.

[28] L. Trefethen, Is Gauss quadrature better than Clenshaw-Curtis? SIAM Review 50 (2008) 67-87.

[29] J. Trinkle, J.S. Pang, S. Sudarsky, G. Lo, On dynamic multi-rigid-body contact problems with Coulomb friction, Journal of Applied Mathematics and Mechanics 4 (1997) 267-279. 